\title{
Computed tomography score and pulmonary function in infants with chronic lung disease of infancy
}

\author{
E.E. Sarria*, R. Mattiello*, L. Rao*, M.R. Wanner", M.E. Raske", C. Tiller*, \\ R. Kimmel* and R.S. Tepper*
}

ABSTRACT: Chronic lung disease of infancy (CLDI) remains a common outcome among infants born extremely prematurely. In older children and adults with lung disease, pulmonary function and computed tomography (CT) scores are used to follow up respiratory disease and assess disease severity. For infants and toddlers, however, these outcomes have been used very infrequently and most often, a dichotomous respiratory outcome (presence or absence of CLDI) is employed.

We evaluated the performance of CT score and pulmonary function to differentiate infants and toddlers with CLDI from a control group.

CT scans, forced expiratory flows and pulmonary diffusing capacity were obtained in 39 CLDI patients and 41 controls (aged 4-33 months). CT scans were quantified using a scoring system, while pulmonary function was expressed as Z-scores. CT score outperformed pulmonary function in identifying those with CLDI. There were no significant correlations between CT score and pulmonary function.

CT score had a better performance than pulmonary function in differentiating individuals with CLDI; however, these outcomes may reflect differing components of the pulmonary pathophysiology of CLDI. This new information on pulmonary outcomes can assist in designing studies with these parameters. Future studies will be required to evaluate which of the outcomes can better detect improvement with therapeutic intervention and/or lung growth.

KEYWORDS: Bronchopulmonary dysplasia, forced expiratory flows, high-resolution computed tomography, lung function, pulmonary diffusing capacity

$\mathbf{T}$ he use of surfactant therapy and improved respiratory care has dramatically increased the survival of infants born extremely prematurely, particularly those born at $<25$ weeks' gestation [1]. While survival following more extreme premature birth has increased, chronic lung disease of infancy (CLDI) or "new" bronchopulmonary dysplasia (BPD) remains a common outcome with long-term respiratory sequelae in older children and adults. There are areas of research that offer exciting potential to minimise lung injury and potentiate lung development following premature birth [2, 3]; however, the assessment of respiratory outcomes for therapeutic interventions, and the natural history of the disease, has been very limited for CLDI and other lung diseases in this very young age group [2]. A dichotomous characterisation of respiratory outcomes (presence or absence) is most often used in CLDI. In older children and adults with lung disease, pulmonary function and computed tomography (CT) scans are used to provide continuous variables, which may not only be more sensitive but may also reflect more directly the underlying pulmonary pathophysiology [4-6].

The raised-volume rapid thoracoabdominal compression technique allows the measurement of adult-type spirometry in sedated infants [7]. Using this technique, investigators have demonstrated that forced expiratory flows are reduced in infants with CLDI [8], comparable to those observed during follow-up of older children with CLDI [9]. In addition, our laboratory has demonstrated that infants and toddlers with CLDI have normal alveolar volumes but reduced pulmonary diffusing capacities [10], which are findings similar to those observed at follow-up of older children born extremely prematurely [11]. Based on the experience of CT scoring systems for cystic fibrosis and chest radiography scores for CLDI, CT scoring systems have been employed in adults and infants to assess lung disease in CLDI survivors $[12,13]$. The purpose of our study was to evaluate, in the same group of infants and toddlers with CLDI, the
AFFILIATIONS

*Section of Pulmonology, and

\#Dept of Radiology, James Whitcomb Riley Hospital for Children, Indiana University, Indianapolis, IN, USA.

CORRESPONDENCE R.S. Tepper

Dept of Pediatrics, Indiana University Medical Center

James Whitcomb Riley Hospital for Children

702 Barnhill Drive

ROC 4270

Indianapolis IN

46202-5225

USA

E-mail: rtepper@iupui.edu

Received:

Nov 042010

Accepted after revision:

March 092011

First published online:

April 082011

European Respiratory Journal

Print ISSN 0903-1936

Online ISSN 1399-3003 
performance of three different state-of-the-art respiratory outcomes for infants: CT score, forced expiratory flow and pulmonary diffusing capacity. These results will provide important information that currently does not exist for designing clinical trials that might select among these different respiratory outcomes for infants with CLDI.

\section{METHODS}

\section{Subjects}

We recruited infants and toddlers from James Whitcomb Riley Hospital for Children (Indianapolis, IN, USA) who were born prematurely (24-29 weeks' gestation) and with a diagnosis of CLDI based upon an oxygen requirement at 28 days' postnatal age or 36 weeks' post-menstrual age [14]. At testing, subjects were clinically stable outpatients with no oxygen requirement and without acute respiratory symptoms for $\geqslant 3$ weeks. Subjects with congenital cardiorespiratory disease were excluded.

For the CT scans, a group of controls who were born at full term (>37 weeks' gestation) were recruited at the Dept of Radiology, James Whitcomb Riley Hospital for Children, from subjects clinically scheduled for a nonpulmonary CT scan under sedation. Subjects were excluded for history of recurrent respiratory illness, wheezing, use of asthma medications, hospitalisation for a respiratory illness or congenital cardiorespiratory abnormalities. Parental consent was obtained for the additional nonclinical CT scan of the chest, and the study was approved by the institutional review board (University of Indiana, Indianapolis, IN, USA).

Control subjects used for pulmonary function had been recruited from advertisements in local publications. These subjects were born at a gestational age of $>37$ weeks, ranged in age between 2 and 26 months, and had no cardiorespiratory malformations, and their respiratory history was negative for wheezing, asthma, treatment with asthma medications or hospitalisation for a respiratory illness.

\section{CT and scoring}

Volumetric CT images were acquired in sedated subjects using an induced respiratory pause at an airway pressure of $20 \mathrm{cmH}_{2} \mathrm{O}$, as previously described $[15,16]$. A low-dose radiation protocol was used ( $\sim 4.8 \mathrm{mGy})$ and patients had proper shielding to decrease the dose to the breast and thyroid. Images were reconstructed using high- and standard-resolution algorithms.

CT images were scored using the system described by OcHIAI et al. [13] for assessing the clinical status of infants with CLDI. The score was calculated for the categories of hyperexpansion, emphysema and fibrous/interstitial abnormalities, and subjective impression. Each category was composed of CT abnormalities found in BPD. The total score ranges $0-18$; higher scores reflect more severe disease. Randomised and deidentified scans were reviewed by two paediatric radiologists blinded to the diagnosis. Each radiologist independently scored the CT scans on two different occasions, with $\geqslant 3$ weeks between scorings.

\section{Infant pulmonary function}

CLDI subjects also had pulmonary function assessed on another day within 1 week of the CT imaging. Subjects were sedated with chloral hydrate $\left(50-75 \mathrm{mg} \cdot \mathrm{kg}^{-1}\right)$. The ratio of diffusing capacity of the lung for carbon monoxide $(D \mathrm{~L}, \mathrm{CO})$ to alveolar volume $(V \mathrm{~A})$ and forced expiratory flows using the raised-volume technique were measured as previously described $[7,17]$. Forced expiratory flow-volume curves were quantified by forced vital capacity (FVC), forced expiratory flows at $50 \%, 75 \%$, and $25-75 \%$ of FVC (FEF50\%, FEF75\%, FEF25$75 \%$, respectively), and forced expiratory volume in $0.5 \mathrm{~s}$ (FEV0.5). The lung function parameters were expressed as Zscores using reference data from our laboratory [7, 17]. Pulmonary function data for CLDI subjects were compared with those from healthy control subjects previously reported from our laboratory [17].

\section{Classification of CLDI severity}

CLDI was classified as mild, moderate or severe using National Institutes of Health (NIH) criteria based upon the requirement for supplemental oxygen at $\geqslant 28$ days' post-natal age or at $\geqslant 36$ weeks' post-menstrual age [14]. CLDI infants breathing room air at $\geqslant 36$ weeks' post-menstrual age were considered as having mild disease, those breathing $<30 \%$ oxygen as having moderate disease and those breathing $>30 \%$ oxygen as having severe disease. Days of supplemental oxygen, days using continuous positive airway pressure (CPAP) and days of mechanical ventilation were quantified from medical records.

\section{Statistical analysis}

CLDI and control groups were compared with statistical tests as appropriate (unpaired t-test, Mann-Whitney U-test and Fisher's exact test). The performance of the CT score and pulmonary function tests for differentiating CLDI from controls was assessed by calculating sensitivity, specificity, likelihood ratios and their corresponding 95\% confidence intervals. Receiver operating characteristic (ROC) curves and the corresponding areas under the curve (AUCs) were calculated. Kappa statistics were used as measures of observer agreement.

Linear regression models were used to assess relationships between CT score, Z-FEF75\% and Z-(DL,CO/VA) and demographics (age, height and sex), and neonatal variables (maternal smoking during pregnancy, gestational age at birth, birth weight, days of CPAP, mechanical ventilation or supplemental oxygen and NIH BPD/CLDI classification). In a backward stepwise multiple linear regression analysis we only included neonatal variables that were significant in the univariate analysis, as there is often co-linearity among these variables. All analyses were performed using SPSS version 18 software (SPSS Inc., Chicago, IL, USA).

\section{RESULTS}

\section{Subjects}

The demographics of our subjects evaluated by CT are summarised in table 1 . There were 39 subjects with CLDI and 41 controls for CT. The majority of the control subjects scheduled for nonchest CT scans were being evaluated for cranial deformities, hearing loss or solid tumours not located in the chest, as previously described [18]. There were no significant differences between the CLDI and control groups in the distributions for sex, race and maternal smoking during pregnancy; however, the CLDI group was younger and smaller in size compared with controls. 


\begin{tabular}{|c|c|c|c|c|}
\hline \multirow[t]{2}{*}{ TABLE 1} & \multicolumn{4}{|c|}{$\begin{array}{l}\text { Demographics for computed tomography } \\
\text { subjects: control versus chronic lung disease of } \\
\text { infancy (CLDI) }\end{array}$} \\
\hline & & Control & CLDI & $p$-value \\
\hline \multicolumn{2}{|l|}{ Subjects } & 41 & 39 & \\
\hline \multicolumn{2}{|l|}{ Females } & $25(61)$ & $21(53)$ & 0.651 \\
\hline \multicolumn{2}{|l|}{ White race } & $29(71)$ & $25(64)$ & 0.635 \\
\hline \multicolumn{2}{|c|}{$\begin{array}{l}\text { Corrected age at } \\
\text { testing months }\end{array}$} & $17(4-33)$ & $12(5-18)$ & 0.002 \\
\hline \multicolumn{2}{|c|}{ Weight at test date $\mathbf{k g}$} & $10.6(6-16)$ & $9.0(5-12)$ & 0.002 \\
\hline \multicolumn{2}{|c|}{ Height at test date $\mathrm{cm}$} & $79.0(59-96)$ & $72.2(58-81)$ & $<0.001$ \\
\hline \multicolumn{2}{|c|}{ Weight/age Z-score } & $0.38(-2-2)$ & $-0.33(-3-2)$ & 0.006 \\
\hline \multicolumn{2}{|c|}{ Length/age Z-score } & $0.08(-2-2)$ & $-1.10(-4-1)$ & $<0.001$ \\
\hline \multicolumn{2}{|c|}{ Gestational age weeks } & $39.1(37-41)$ & 25.5 (23-29) & $<0.001$ \\
\hline \multicolumn{2}{|c|}{ Birth weight kg } & $3.4(2.7-4.1)$ & $0.87(0.49-1.44)$ & $<0.001$ \\
\hline \multicolumn{2}{|c|}{$\begin{array}{l}\text { Maternal smoking } \\
\text { during pregnancy }\end{array}$} & $11(27)$ & $10(26)$ & 1.00 \\
\hline \multicolumn{2}{|c|}{$\begin{array}{l}\text { Mechanical ventilation } \\
\text { days }\end{array}$} & & $26(0-83)$ & \\
\hline \multicolumn{2}{|c|}{ Use of oxygen days } & \multicolumn{3}{|c|}{$86.2(28-170)$} \\
\hline \multicolumn{2}{|c|}{ CPAP days } & \multicolumn{3}{|c|}{$19.0(0-50)$} \\
\hline \multicolumn{5}{|c|}{ NIH BPD/CLDI severity } \\
\hline \multicolumn{2}{|c|}{ Mild } & \multicolumn{3}{|c|}{$11(28)$} \\
\hline \multicolumn{2}{|l|}{ Moderate } & \multicolumn{3}{|c|}{$5(12)$} \\
\hline \multicolumn{2}{|l|}{ Severe } & \multicolumn{3}{|c|}{$23(59)$} \\
\hline
\end{tabular}

Data are presented as $n, n$ (\%) or mean (range), unless otherwise stated. CPAP: continuous positive airway pressure; $\mathrm{NIH}$ : National Institutes of Health; BPD: broncopulmonary dysplasia.

\section{CT score}

The three most frequent abnormal CT findings for the CLDI group were triangular subpleural opacities (89\%), mosaic pattern of lung attenuation (82\%) and distortion or thickening of the bronchovascular bundle (79\%) (table 2). The interobserver agreement for the total CT score had a kappa value of 0.71 , while the different categories had kappa values up to 0.76 . The intraobserver kappa values were 0.85 for the total score and 0.77-0.95 for the different categories.

Total CT score was significantly higher for CLDI than control subjects ( 9.15 versus $0.93 ; \mathrm{p}<0.001)$. The scores for each category were also significantly greater for CLDI than for control subjects ( $\mathrm{p}<0.001$; hyperexpansion 2.82 versus 0.39 ; emphysema 1.77 versus 0.15 ; fibrous/interstitial abnormalities 3.54 versus 0.39 ; subjective 1 versus 0.05 ). In univariate analysis, total CT score was inversely correlated with gestational age at birth; infants with lower gestational age had higher CT scores or worse disease $\left(\beta=-1.27 ; r^{2}=0.43, p=0.007\right)$. In addition, higher $\mathrm{CT}$ scores were correlated with a greater number of days of mechanical ventilation $\left(\beta=0.12 ; \mathrm{r}^{2}=0.58, \mathrm{p}<0.001\right)$, greater number of days of supplemental oxygen $\left(\beta=0.003 ; \mathrm{r}^{2}=0.61\right.$, $\mathrm{p}<0.001)$, and a higher NIH severity classification $(\beta=2.58$; $\left.\mathrm{r}^{2}=0.46, \mathrm{p}=0.003\right)$. When analysed by stepwise multiple regression analysis, only increasing days of oxygen was significantly associated with higher total CT score (table 3).

\begin{tabular}{|c|c|c|}
\hline CT score findings & CLDI & Control \\
\hline Hyperexpansion & $28(71)$ & $1(2)$ \\
\hline Mosaic pattern of lung attenuation & $32(82)$ & $0(0)$ \\
\hline Intercostal bulging & $20(51)$ & $0(0)$ \\
\hline Bullae or blebs & $20(51)$ & $2(5)$ \\
\hline Triangular subpleural opacities & $35(89)$ & $9(22)$ \\
\hline $\begin{array}{l}\text { Distortion and thickening of bronchovascular } \\
\text { bundle }\end{array}$ & $31(79)$ & $4(9)$ \\
\hline Consolidation & $28(71)$ & $0(0)$ \\
\hline
\end{tabular}

Data are presented as $\mathrm{n}(\%)$

A total CT score $>2$ had a sensitivity and specificity of $87 \%$, a likelihood ratio of 7 and an AUC of 0.94. The performances for each of the categories used in the total CT score are summarised in table 4 . Fibrous/interstitial abnormalities was the individual category with the highest AUC.

\section{Pulmonary function}

The CLDI group had significantly lower forced expiratory flows, but not FVC or FEV0.5, compared with healthy subjects evaluated in our laboratory (table 5). In univariate analysis, ZFEF75\% was inversely correlated with maternal smoking during pregnancy. Infants whose mothers smoked during pregnancy had lower forced expiratory flows $\left(\beta=-0.88 ; r^{2}=0.34, p=0.035\right)$. In addition, Z-FEF75\% was inversely correlated with increasing days of using CPAP in the neonatal period; infants who used $\mathrm{CPAP}$ for more days had lower forced expiratory flows $\left(\beta=-0.03 ; r^{2}=0.34, p=0.035\right)$. Using stepwise multiple regression analysis, both maternal smoking during pregnancy and days of CPAP remained independently associated with lower ZFEF75\% (table 3). CLDI subjects also had significantly lower values for $D \mathrm{~L}, \mathrm{CO} / V \mathrm{~A}$ compared with healthy subjects evaluated in our laboratory (table 3). Z-DL,CO/VA was inversely correlated with corrected age at time of evaluation; older subjects had

\begin{tabular}{llll}
\hline TABLE 3 & $\begin{array}{l}\text { Multivariate analysis of total computed } \\
\text { tomography (CT) score with chronic lung } \\
\text { disease of infancy demographics }\end{array}$ & \\
& $\boldsymbol{\beta}(\mathbf{9 5 \%} \mathbf{C l})$ & p-value & $\mathbf{r}^{\mathbf{2}}$ \\
\hline $\begin{array}{l}\text { CT Score } \\
\text { Use of } \mathrm{O}_{2} \text { days }\end{array}$ & $0.083(0.047-0.118)$ & $<0.001$ & 0.611 \\
$\begin{array}{l}\text { M-FEF75\% } \\
\text { CPAP days }\end{array}$ & $-0.983(-1.742--0.225)$ & 0.013 & 0.513 \\
$\begin{array}{l}\text { Z-DL,co/VA } \\
\text { Corrected age months }\end{array}$ & $-0.036(-0.064--0.08)$ & 0.013 & \\
\hline
\end{tabular}

Z: Z-score; FEF75\%: forced expiratory flow at $75 \%$ of forced vital capacity; CPAP: continuous positive airway pressure; DL,CO: diffusing capacity of the lung for carbon monoxide; VA: alveolar volume. 


\begin{tabular}{|c|c|c|c|c|c|c|}
\hline \multirow{2}{*}{$\begin{array}{l}\text { TABLE } 4 \\
\text { Parameter }\end{array}$} & \multicolumn{6}{|c|}{$\begin{array}{l}\text { Diagnostic performance of computed tomography score for differentiating subjects with chronic lung disease of } \\
\text { infancy from controls }\end{array}$} \\
\hline & & Criteria & Sensitivity \% & Specificity \% & +LR & AUC \\
\hline \multicolumn{7}{|c|}{ Score category } \\
\hline \multicolumn{2}{|c|}{ Hyperexpansion } & $>1$ & $71(55-85)$ & 95 (83-99) & $14(11-18)$ & $0.88(0.79-0.94)$ \\
\hline \multicolumn{2}{|c|}{ Emphysema } & $>0$ & $51(34-67)$ & 95 (83-99) & $10(7-14)$ & $0.73(0.62-0.83)$ \\
\hline \multicolumn{2}{|c|}{ Subjective impression } & $>0$ & $71(55-85)$ & 95 (83-99) & $14(11-18)$ & $0.84(0.74-0.91)$ \\
\hline \multicolumn{2}{|l|}{ Total score } & $>2$ & 87 (72-95) & 87 (73-95) & 7 (6-8) & $0.94(0.86-0.98)$ \\
\hline
\end{tabular}

Data are presented with 95\% confidence intervals. +LR: positive likelihood ratio; AUC: area under the curve.

lower pulmonary diffusion $\left(\beta=-0.115 ; \mathrm{r}^{2}=0.37, \mathrm{p}=0.021\right)$ The stepwise multiple regression analyses are displayed in table 3.

Using a cut-off of Z-score $\leqslant-2.0$ ( 2 SD below the mean for healthy controls), the performance of the pulmonary function parameters is summarized in table $6 . D \mathrm{~L}, \mathrm{CO} / V \mathrm{~A}$ had the best performance, while the forced expiratory flow parameters had similar, but lower performance than $D \mathrm{~L}, \mathrm{CO} / V \mathrm{~A}$.

Comparison of ROC curves for total CT score, Z-DL,CO/VA and Z-FEF75\% to differentiate CLDI from controls are illustrated in figure 1 . Total CT score had the best performance, followed by Z-DL,CO/VA and then Z-FEF75\%.

\section{DISCUSSION}

Our study demonstrated that a CT scoring system for images obtained using the controlled ventilation technique performs well in discriminating infants and toddlers with CLDI from those without lung disease. Among the subjects with CLDI, the total CT score correlated best with days of supplemental oxygen. In addition, total CT score outperformed measurements of pulmonary function. A major strength of this study was that several state-of-the-art respiratory outcomes were evaluated in the same group of infants with CLDI and that for each outcome, we had control data obtained at the same institution with the same methodology.

\begin{tabular}{lcccc}
\hline TABLE 5 & Pulmonary function data & \\
Parameter & CLDI & Control & $\begin{array}{c}\text { 95\% CI of } \\
\text { CLDI-control }\end{array}$ & p-value \\
& & & & \\
\hline Z-FVC & $-0.04 \pm 0.95$ & $0.06 \pm 1.32$ & $-0.357-0.582$ & 0.636 \\
Z-FEV0.5 & $-0.38 \pm 0.79$ & $-0.12 \pm 1.28$ & $-0.184-0.708$ & 0.247 \\
Z-FEF5\% & $-0.68 \pm 0.97$ & $-0.29 \pm 1.15$ & $-0.035-0.812$ & 0.072 \\
Z-FEF75\% & $-0.68 \pm 1.14$ & $-0.04 \pm 1.19$ & $0.196-1.098$ & 0.005 \\
Z-FEF25-75\% & $-0.80 \pm 1.02$ & $-0.29 \pm 1.23$ & $0.629-0.961$ & 0.026 \\
Z-DL,Co/VA & $-0.92 \pm 1.10$ & $-0.00 \pm 0.98$ & $0.401-0.942$ & $<0.001$ \\
\hline
\end{tabular}

Data are presented as mean $\pm \mathrm{SD}$, unless otherwise stated. CLDI: chronic lung disease of infancy; Z: Z-score; FVC: forced vital capacity; FEV0.5: forced expiratory volume in $0.5 \mathrm{~s}$; FEF50\%: forced expiratory flow at $50 \%$ of FVC; FEF75\%: forced expiratory flow at $75 \%$ of FVC; FEF25-75\%: forced expiratory flow at $25-75 \%$ of FVC; DL,CO: diffusing capacity of the lung for carbon monoxide; VA: alveolar volume.
The two paediatric radiologists participating in this study had no previous experience with the CT scoring system employed; however, we found good intra- and interobserver agreement, comparable to values reported in other studies $[6,13,19]$. The distribution of abnormalities in our CLDI subjects was also consistent with previous descriptions of CT findings in subjects with CLDI [12, 13, 20-22]. A detailed comparison of CT abnormalities in our study and those from other studies is difficult, owing to differences in methods used to obtain the $\mathrm{CT}$, age of subjects evaluated, severity of disease and new versus old BPD. Nevertheless, it is important to note that we found triangular subpleural opacities, which is a common abnormality reported in other studies, in almost $90 \%$ of our CLDI subjects. This finding, which probably reflects minimal dependent atelectasis seen in sedated infants, was also the most frequent abnormality reported for our control subjects and emphasizes the need for control subjects.

We found good sensitivity and specificity for the total CT score. A total score $>2$ had a positive likelihood ratio of 7 and an AUC of 0.94, while sensitivity and specificity for the individual components of the total score were also high. There were significant correlations between total CT score and several neonatal parameters. Increasing CT score was related

TABLE 6 Performance of lung function parameters in differentiating subjects with chronic lung disease of infancy from controls

\begin{tabular}{lccccc} 
Parameter & Criteria & Sensitivity $\%$ & Specificity $\%$ & + LR & AUC \\
\hline Z-FVC & $\leqslant-2.0$ & $3(0.1-14)$ & $98(92-99)$ & $1(0.2-8)$ & $0.56(0.47-0.65)$ \\
Z-FEV 0.5 & $\leqslant-2.0$ & $3(0.1-13)$ & $97(92-99)$ & $1(0.2-8)$ & $0.61(0.52-0.70)$ \\
Z-FEF50\% & $\leqslant-2.0$ & $5(3-25)$ & $96(89-99)$ & $1(0.3-5)$ & $0.62(0.53-0.71)$ \\
Z-FEF75\% & $\leqslant-2.0$ & $13(4-28)$ & $92(85-97)$ & $2(0.7-4)$ & $0.68(0.59-0.76)$ \\
Z-FEF25-75\% & $\leqslant-2.0$ & $10(3-25)$ & $92(85-97)$ & $2(0.7-4)$ & $0.65(0.56-0.73)$ \\
Z-DL,CO/VA & $\leqslant-2.0$ & $26(13-43)$ & $98(90-100)$ & $14(8-23)$ & $0.78(0.68-0.86)$
\end{tabular}

Data are presented as quantity or quantity $(95 \% \mathrm{Cl}) .+\mathrm{LR}$ : positive likelihood ratio; AUC: area under the curve; Z: Z-score; FVC: forced vital capacity; FEV0.5 forced expiratory volume in $0.5 \mathrm{~s}$; FEF50\%: forced expiratory flow at $50 \%$ of FVC FEF75\%: forced expiratory flow at 75\% of FVC; FEF25-75\%: forced expiratory flow at $25-75 \%$ of FVC; DL,CO: diffusing capacity of the lung for carbon monoxide; VA: alveolar volume. 


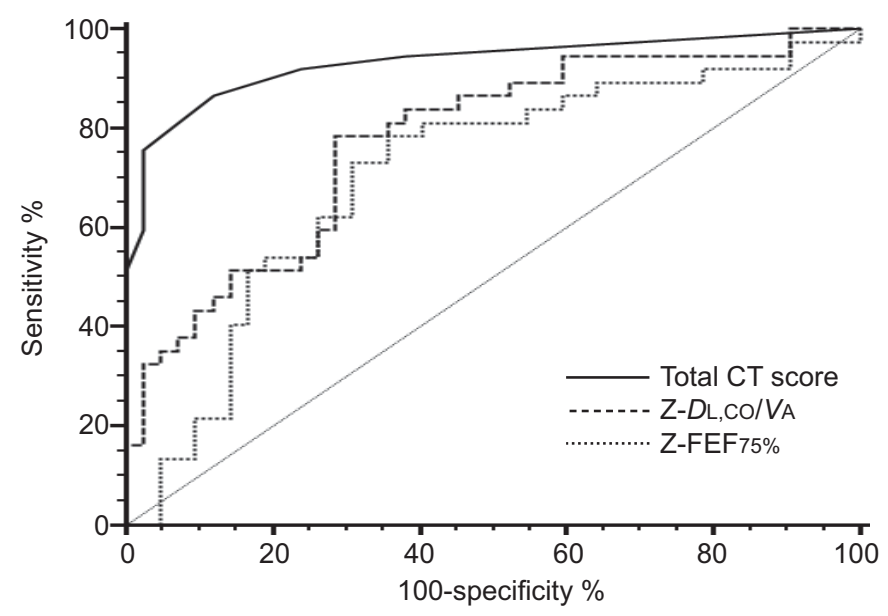

FIGURE 1. Receiver operating characteristic curves for total computed tomography (CT) score, diffusing capacity of the lung for carbon monoxide/ alveolar volume Z-score (Z-DL,CO/VA) and forced expiratory flow at $75 \%$ of forced vital capacity Z-score (Z-FEF75\%) in differentiating subjects with chronic lung disease of infancy from controls.

to greater prematurity at birth (lower gestational age at birth), as well as more days with mechanical ventilation or supplemental oxygen. As many of these neonatal parameters are highly correlated, multivariate analysis indicated that only days of supplemental oxygen in the newborn intensive care unit remained significant. This finding is consistent with the NIH BPD/CLDI scoring system, which uses days of oxygen to define severity of disease [14].

Our measures of pulmonary function demonstrated lower pulmonary diffusing capacity and lower forced expiratory flows in the CLDI group. These findings are consistent with the pulmonary pathophysiology of CLDI, which includes airway and parenchymal disease [9, 10, 23-25]. Among our CLDI subjects, maternal smoking was associated with lower forced expiratory flows, a finding that extends to this population of premature infants the adverse effects of maternal smoking on lung function that have previously been reported for full-term infants $[7,26]$. As a group, CLDI patients had significantly lower pulmonary function than controls; however, measures of pulmonary function were not as good as total CT score in distinguishing CLDI from control subjects. This finding probably reflects the greater inter-subject biological variability of physiological measurements compared to the intersubject variability of CT scores among control subjects.

We did not find a significant correlation between pulmonary function and CT score among infants and toddlers with CLDI $(\mathrm{r}=-0.07-0.13)$. This contrasts with the small, but statistically significant relationship between increased parenchymal opacities and lower functional residual capacity among infants with BPD in a study by MAHUT et al. [21]. However, in that study, functional residual capacity and CT scans were obtained in sedated infants during tidal breathing, which is frequently associated with the development of atelectasis in supine sedated infants [27], which may have accounted for the weak correlation they observed. In our study, the absence of a correlation between CT score and physiological measurements suggests that these measurements offer differing assessments of the lung and may offer complimentary, rather than correlative information. Total CT score had the best overall ROCs with the highest AUC. Although the AUCs were similar for the several measures of pulmonary function, $D L, C O / V A$ had a higher specificity and likelihood ratio than the forced expiratory flows, which may reflect the fact that CLDI is more a chronic disease of the lung parenchyma than the airways.

There are several limitations to our study. Currently, there is no generally accepted CT scoring system for infants with CLDI. AUKLAND and co-workers $[12,28]$ have used a modified Bhalla score in "old" BPD survivors, which included a detailed scoring system that has only been used for older children and young adults with BPD, not infants with CLDI. The scoring system proposed by OCHIAI et al. [13] has not been used by other investigators; however, it was developed for infants in the "new" BPD era. In addition, these investigators found signicant relationships between CT score and neonatal demographics, which led us to use it in our study. Our control CT group cannot be characterised as completely normal, as subjects had a nonrespiratory medical problem that led their physician to schedule a nonchest CT scan. However, we excluded from our control group subjects with current or prior history of respiratory problems and their lung scans were considered normal by a paediatric radiologist. Therefore, we believe that our subjects represent good controls for CLDI subjects and should not have affected the interpretation of our results. Also, our CT control subjects did not undergo pulmonary function measurements, as we would not have been successful in recruiting them if they had to return for an additional visit for pulmonary function testing. Therefore, the same controls were not used for CT and pulmonary function measurements; however, the pulmonary function of healthy controls had previously been evaluated in our laboratory using the same equipment and technique. Lastly, our subjects with CLDI included a cross-sectional group with ages $5-18$ months who were clinically stable outpatients. These subjects were not part of a cohort and therefore do not represent the full distribution of disease severity. As the methodologies used to assess pulmonary function and to obtain the CT scans require sedation, we did not evaluate subjects with the most severe disease or when they were diagnosed with CLDI in the newborn intensive care unit. However, our subjects reflect the majority of patients that survive with CLDI and we included a range of severity that might well be reflected in future clinical trials. Our results will provide important information for designing clinical trials that may use the respiratory outcomes evaluated in the current study.

In conclusion, CT score demonstrated good performance in differentiating infants and toddlers with CLDI and correlated with neonatal and clinical variables. The $\mathrm{CT}$ score outperformed the pulmonary function tests and there were no significant correlations between the two outcomes, which may reflect differing components of the CLDI pulmonary pathophysiology. Although CT score appears to provide a good quantitative assessment for CLDI, it remains unclear whether CT scores or pulmonary function can better assess changes in respiratory status following a therapeutic intervention or identify improvements with lung growth and development. There are no current data suggesting that these outcomes are useful in the clinical management of infants with CLDI. While sedation is required to 
obtain all of these outcomes, the relative risks associated with each will need to be considered, particularly the exposure to ionising radiation. The new information on pulmonary outcomes provided by this study can assist in designing future research studies with these parameters as outcomes.

\section{SUPPORT STATEMENT}

The present study was supported by the National Institutes of Health (Bethesda, MD, USA), grant number HL054062.

\section{STATEMENT OF INTEREST}

None declared.

\section{REFERENCES}

1 Kinsella JP, Greenough A, Abman SH. Bronchopulmonary dysplasia. Lancet 2006; 367: 1421-1431.

2 Castro M, Ramirez MI, Gern JE, et al. Strategic plan for pediatric respiratory diseases research: an NHLBI Working Group report. Proc Am Thorac Soc 2009; 6: 1-10.

3 Warburton D, Perin L, Defilippo R, et al. Stem/progenitor cells in lung development, injury repair, and regeneration. Proc Am Thorac Soc 2008; 5: 703-706.

4 Davis SD, Brody AS, Emond MJ, et al. Endpoints for clinical trials in young children with cystic fibrosis. Proc Am Thorac Soc 2007; 4: 418-430.

5 Davis SD, Rosenfeld M, Kerby GS, et al. Multicenter evaluation of infant lung function tests as cystic fibrosis clinical trial endpoints. Am J Respir Crit Care Med 2010; 182: 1387-1397.

6 Mattiello R, Sarria E, Mallol J, et al. Post-infectious bronchiolitis obliterans: can CT scan findings at early age anticipate lung function? Pediatr Pulmonol 2010; 45: 315-319.

7 Jones M, Castile R, Davis S, et al. Forced expiratory flows and volumes in infants - normative data and lung growth. Am J Respir Crit Care Med 2000; 161: 353-359.

8 Robin B, Kim YJ, Huth J, et al. Pulmonary function in bronchopulmonary dysplasia. Pediatr Pulmonol 2004; 37: 236-242.

9 Fawke J, Lum S, Kirkby J, et al. Lung function and respiratory symptoms at 11 years in extremely preterm children: the EPICure Study. Am J Respir Crit Care Med 2010; 182: 237-245.

10 Balinotti JE, Chakr VC, Tiller C, et al. Growth of lung parenchyma in infants and toddlers with chronic lung disease of infancy. Am J Respir Crit Care Med 2010; 181: 1093-1097.

11 Welsh L, Kirkby J, Lum S, et al. The EPICure study: maximal exercise and physical activity in school children born extremely preterm. Thorax 2010; 65: 165-172.

12 Aukland SM, Halvorsen T, Fosse KR, et al. High-resolution CT of the chest in children and young adults who were born prematurely: findings in a population-based study. AJR Am J Roentgenol 2006; 187: 1012-1018.
13 Ochiai M, Hikino S, Yabuuchi $\mathrm{H}$, et al. A new scoring system for computed tomography of the chest for assessing the clinical status of bronchopulmonary dysplasia. J Pediatr 2008; 152: 90-95.

14 Jobe AH, Bancalari E. Bronchopulmonary dysplasia. Am J Respir Crit Care Med 2001; 163: 1723-1729.

15 Llapur CJ, Martinez TM, Coates C, et al. Lung structure and function of infants with recurrent wheeze when asymptomatic. Eur Respir J 2009; 33: 107-112.

16 Martinez TM, Llapur CJ, Williams TH, et al. High-resolution computed tomography imaging of airway disease in infants with cystic fibrosis. Am J Respir Crit Care Med 2005; 172: 1133-1138.

17 Balinotti JE, Tiller CJ, Llapur CJ, et al. Growth of the lung parenchyma early in life. Am J Respir Crit Care Med 2009; 179: 134-137.

18 Rao L, Tiller C, Coates C, et al. Assessment of airway growth invivo using high resolution computed tomography in infants and toddler. Am J Respir Crit Care Med 2009; 179: A3280.

19 de Jong PA, Ottink MD, Robben SG, et al. Pulmonary disease assessment in cystic fibrosis: comparison of $\mathrm{CT}$ scoring systems and value of bronchial and arterial dimension measurements. Radiology 2004; 231: 434-439.

20 Howling SJ, Northway WH Jr, Hansell DM, et al. Pulmonary sequelae of bronchopulmonary dysplasia survivors: high-resolution CT findings. AJR Am J Roentgenol 2000; 174: 1323-1326.

21 Mahut B, de Blic J, Emond S, et al. Chest computed tomography findings in bronchopulmonary dysplasia and correlation with lung function. Arch Dis Child Fetal Neonatal Ed 2007; 92: F459-F464.

22 Oppenheim C, Mamou-Mani T, Sayegh N, et al. Bronchopulmonary dysplasia: value of $\mathrm{CT}$ in identifying pulmonary sequelae. AJR Am J Roentgenol 1994; 163: 169-172.

23 Fakhoury KF, Sellers C, Smith EOB, et al. Serial measurements of lung function in a cohort of young children with bronchopulmonary dysplasia. Pediatrics 2010; 125: e1441-e1447.

24 Narang I, Bush A, Rosenthal M. Gas transfer and pulmonary blood flow at rest and during exercise in adults 21 years after preterm birth. Am J Respir Crit Care Med 2009; 180: 339-345.

25 Narang I, Rosenthal M, Cremonesini D, et al. Longitudinal evaluation of airway function 21 years after preterm birth. Am J Respir Crit Care Med 2008; 178: 74-80.

26 Stocks J, Dezateux C. The effect of parental smoking on lung function and development during infancy. Respirology 2003; 8: 266-285.

27 Lutterbey G, Wattjes MP, Doerr D, et al. Atelectasis in children undergoing either propofol infusion or positive pressure ventilation anaesthesia for magnetic resonance imaging. Paediatr Anaesth 2007; 17: 121-125.

28 Aukland SM, Rosendahl K, Owens CM, et al. Neonatal bronchopulmonary dysplasia predicts abnormal pulmonary HRCT scans in long-term survivors of extreme preterm birth. Thorax 2009; 64: 405-410. 